\title{
ORIGINAL ARTICLE \\ Documentation of weight management practices for individuals with spinal cord injuries and disorders
}

\author{
SM Locatelli ${ }^{1,2}$ and SL LaVela ${ }^{1,2,3}$
}

Study design: This cross-sectional chart review study included 100 US Veterans with spinal cord injuries/disorders (SCI/D) who received care at a Veterans Affairs (VA) SCl facility during a 12-month period. Progress notes were examined to extract need for weight management (WM), patient-provider discussions about risk due to overweight/obesity, recommended lifestyle changes and/or follow-up and WM education.

Objectives: To understand what WM services are offered to Veterans with SCI/D within the VA SCl System of Care during comprehensive preventive health evaluations (annual evaluations), inpatient stays and outpatient visits.

Setting: VA SCI System of Care, Department of Veterans Affairs, United States.

Results: Overall, 73\% demonstrated a need for WM. Weight was most frequently addressed during the nutrition assessment of annual evaluations, but this assessment was most likely to be skipped. Nutrition histories were missing many key components. Over half received WM education; individuals who were described as overweight/obese by their provider were more likely to receive education. Most of the Veterans who were seen in an inpatient setting were weighed; weight was only discussed with $12 \%$. Less than half of the Veterans with outpatient visits were weighed, and 23\% received WM recommendations.

Conclusions: Weight was frequently discussed during nutrition assessments, but infrequently addressed during outpatient or inpatient encounters. Few Veterans received specific recommendations on caloric/nutrient requirements and nutrition histories were missing recommended elements. Additional work is needed to help providers to incorporate WM information into care.

Spinal Cord (2016) 54, 1176-1182; doi:10.1038/sc.2016.59; published online 10 May 2016

\section{INTRODUCTION}

Over $70 \%$ of individuals with spinal cord injuries and disorders (SCI/D) are overweight or obese, based on body mass index (BMI). ${ }^{1,2}$ Being overweight/obese increases risk for a variety of health consequences. For instance, in the general population, overweight/obesity increases risk for weight-related comorbidities, such as diabetes mellitus, hypertension and dyslipidemia. ${ }^{3}$ Individuals with SCI/D also have additional potential health consequences of overweight/obesity, including functional impairments, such as difficulty ambulating or transferring, ${ }^{4}$ and increased risk for pressure ulcer, ${ }^{5}$ pain ${ }^{6}$ and fractures. ${ }^{7}$ As such, weight management (WM) is essential among individuals with SCI/D to preserve health and quality of life.

Individuals with SCI/D experience multiple barriers to $\mathrm{WM}$, including barriers directly related to their SCI/D or related to secondary conditions, as well as indirect barriers. Direct barriers include physical limitations inhibiting physical activity and decreased metabolism. Multiple studies of barriers to WM have identified difficulty in exercising ${ }^{8}$ and fatigue/lack of energy as common barriers. ${ }^{8,9}$ Individuals with SCI/D also have lower basal metabolic rate than the general population because of differences in muscle mass and sympathetic nervous system activity; even when compared with able-bodied persons with similar energy expenditure, basal metabolic rates among individuals with SCI/D are $30-75 \%$ of basal metabolic rates of able-bodied persons. ${ }^{10}$
Indirect barriers that affect WM include issues with access, lack of knowledge of safe exercises and limitations on healthy food options. Lack of places to exercise has been reported as one of the most common experienced barriers among individuals with SCI/D. ${ }^{8}$ Cowan et al. ${ }^{9}$ found that a little more than half of participants with SCI/D knew of an accessible fitness center and felt welcome at such a center; however, only $12 \%$ believed an exercise instructor at a typical fitness center would know how to adapt an exercise program for SCI/D. Individuals with SCI/D may also lack knowledge of suitable and safe exercises $^{11}$ that research suggests is a frequently reported barrier. ${ }^{9}$ Finally, individuals with SCI/D may have limitations on healthy food choices because of difficulty in traveling to restaurants or stores with healthier options or because they are not responsible for preparing meals. $^{12}$

Providers have also reported barriers to addressing overweight/ obesity and WM with individuals with SCI/D. Providers have shared that they feel discomfort with discussing WM with Veterans with SCI/D, and believed in some cases competing medical needs took precedence. ${ }^{12}$ In addition, the lack of evidence-based care guidelines on WM among individuals with SCI/D also impedes providers from offering WM guidance. ${ }^{12}$ One study found that only $22 \%$ of individuals with SCI/D received specific information about SCI-appropriate exercises from a physician, although more than half said their physician had recommended exercise. ${ }^{9}$

${ }^{1}$ Center of Innovation for Complex Chronic Healthcare, Hines VA Hospital, Department of Veterans Affairs (DVA), Hines, IL, USA; ${ }^{2}$ Spinal Cord Injury Quality Enhancement Research Initiative, Hines VA Hospital, DVA, Hines, IL, USA and ${ }^{3}$ Center for Healthcare Studies, Feinberg School of Medicine, Northwestern University, Chicago, IL, USA Correspondence: Dr SM Locatelli, Hines VA Hospital, Health Services Research \& Development, 5000 South 5th Avenue (Mailcode 151H), Hines, IL 60141, USA.

E-mail: Sara.Locatelli@va.gov

Received 30 September 2015; revised 19 February 2016; accepted 7 March 2016; published online 10 May 2016 
Furthermore, providers have reported concerns with using BMI as an indicator of overweight/obesity among individuals with SCI/D. ${ }^{12}$ Although BMI is a frequently used indicator, it may explain less variance in percent body fat in individuals with SCI/D than the general population. ${ }^{13}$ Laughton et al. ${ }^{14}$ examined BMI and percent body fat, and found that the standard BMI obesity cutoff of $30 \mathrm{~kg} \mathrm{~m}^{-2}$ failed to identify $73.9 \%$ of individuals with SCI/D who were obese based on percent body fat. They recommend using a lower BMI cutoff of $25 \mathrm{~kg} \mathrm{~m}^{-2}$ to indicate obesity, and $22 \mathrm{~kg} \mathrm{~m}^{-2}$ to identify overweight individuals at risk of developing weight-related conditions and in need of further screening.

In the Veterans Health Administration (VHA), WM education is delivered, in part, through MOVE! that provides group classes and individual consults with physicians, dietitians, psychologists and/or therapists to reach weight loss/maintenance goals. ${ }^{15,16}$ As the MOVE! WM program is intended for Veterans in general, Veterans with SCI/D would also be able to obtain WM education and treatment through this program. However, $<0.02 \%$ of Veterans with SCI/D have used MOVE! services (M-W Sohn, unpublished data, VA HSR\&D, 11/2009). In addition, research with providers suggests Veterans with SCI/D are hesitant to use MOVE! because of non-SCI/D-specific information and discomfort attending sessions with able-bodied Veterans, and prefer to instead receive WM care through the Veterans Affairs (VA) Spinal Cord Injury (SCI) System of Care. ${ }^{12}$ SCI/D providers were also hesitant to recommend MOVE! because of lack of knowledge about the program and belief that the information would not be relevant for individuals with SCI/D. ${ }^{12}$

The purpose of the present study was to understand what WM services are being offered to Veterans with SCI/D, including what is done on a patient-by-patient basis during annual evaluations, inpatient stays and outpatient encounters.

\section{MATERIALS AND METHODS}

\section{Settings and participants}

The VA SCI System of Care uses a 'hub and spoke' model. ${ }^{17}$ Comprehensive primary and specialty care for Veterans with SCI/D is provided at SCI Centers (hubs), typically located at large VA medical centers, that have multidisciplinary care teams. ${ }^{17}$ SCI spoke sites have dedicated SCI primary care teams, and are required to have at least four team members, including a physician, registered nurse care manager, social worker and clinical associate. ${ }^{17}$ Veterans with SCI/D are referred to the nearest SCI Center to receive postinjury rehabilitation, inpatient care and annual comprehensive preventive health evaluations (also known as annual evaluations (AEs)) that may be conducted on an inpatient or outpatient basis. ${ }^{18}$ Although the AE includes up to 21 components, such as urinary tract evaluation and pulmonary function, ${ }^{18}$ in the present study, we focused on four assessments most relevant to WM: cardiovascular; psychological, social and vocational (psychosocial); comprehensive rehabilitation functional; and dietary and nutritional.

Using administrative data (VA Spinal Cord Injury and Disorders Outcomes database), we identified Veterans with SCI/D who had been seen at least once in the previous 12 months at one of 3 VA SCI facilities. A retrospective chart review was conducted in which progress notes, outpatient visits and inpatient stays over a 12-month period (1 November 2012 to 31 October 2013) were examined to gather both quantitative and qualitative data on WM practices for Veterans with SCI/D. This study was reviewed and approved by the local institutional review board; we certify that all applicable institutional and governmental regulations concerning the ethical use of human volunteers were followed during the course of this research. As this study only involved chart review data, no informed consent or Health Information Portability and Accountability Act (HIPAA) authorization were necessary.

\section{Measures}

To ensure consistent and accurate data collection across patients, a structured chart review form was developed and used. This chart review form was reviewed by SCI providers and was pilot-tested on 10 randomly selected charts. The following information was extracted from charts: indicators of need for $\mathrm{WM}, \mathrm{WM}$ during AEs, WM education received outside of $\mathrm{AE}$, WM during non- $\mathrm{AE}$ inpatient care and $\mathrm{WM}$ during non- $\mathrm{AE}$ outpatient care. The chart review form also included demographics: race, ethnicity, gender, date of birth and marital status; and SCI characteristics: American Spinal Injury Association (ASIA) impairment score, level of injury (paraplegia vs tetraplegia, specific level of injury if available), date of injury and etiology (traumatic vs nontraumatic).

Indicators of need for WM included most recent height, weight and BMI (and date of these measures), percent of ideal body weight and percent body fat. Diagnoses of overweight and obesity were coded based on International Classification of Diseases, Ninth Revision (ICD-9) diagnosis codes to ensure consistency in coding overweight/obesity diagnosis: V77.8 Obesity, 277.7 Dysmetabolic syndrome, 278.0 Overweight and obesity (with the following subcategories coded as appropriate: 278.01 Morbid/Severe obesity, 278.02 Overweight), 278.1 Localized adiposity and 783.1 Abnormal weight gain. In addition, the following weight-related comorbidities were coded, once again using ICD-9 codes to ensure consistency across cases: 250.0-250.9 Diabetes mellitus, 272.0-272.9 Dyslipidemia, 327.2 Sleep apnea, 401.0-401.9 Hypertension and 715.0 Degenerative joint disease. Finally, recent changes in weight, changes in functional status, discussion of interest in WM and description of the patient as overweight or obese (by the primary care provider) were coded based on progress notes.

Results of the most recent $\mathrm{AE}$ were also extracted from the patient chart. For all cases, date of the $\mathrm{AE}$ (or dates, if it spanned multiple days) and whether it was performed on an inpatient or outpatient basis were coded. Information was also coded on completion of four assessments: cardiovascular, psychosocial, rehabilitation and nutrition. For each assessment, the following information was obtained: provider type completing the assessment (for example, physician, nurse practitioner, psychologist), whether weight was discussed as a risk factor, recommendations for lifestyle changes to reduce risk (healthy diet, physical activity/exercise and weight loss), recommendations for follow-up WM care (for example, follow-up appointment with provider, referral) and delivery of WM education. Furthermore, for the nutrition assessment, details of the nutrition history were coded, based on the recommended necessary components of a full nutrition history identified in the VHA Clinical Nutrition Management Handbook. ${ }^{19}$ Recommendations on medical nutrition therapy, discussion of caloric or nutrient requirements, diet prescription, goal setting with regard to healthy eating or WM (for example, tracking losing, or maintaining weight) and initiating follow-up at defined intervals were also extracted.

Information on inpatient stays and outpatient encounters with primary care providers (physician, physician's assistant, nurse practitioner), dietitians, psychologists/social workers and physical therapists were also coded. For inpatient stays, the following were coded: number of inpatient days, whether the patient was weighed and method used (for example, wheelchair scale, Hoyer lift), whether weight was discussed as a concern, whether plans were made for postdischarge WM, recommendations for WM (healthy diet, physical activity/exercise, weight loss) and referrals to WM professionals or programs. Number of meetings with dietitians, psychologists/social workers and therapists were extracted, and weight-related discussions during these meetings were summarized. Similar information was obtained for outpatient encounters (for example, whether patient was weighed and how, recommendations, referrals).

Finally, information on WM education delivered outside of AEs (for example, during an inpatient stay for a medical reason) was coded, including date and title of education, type of provider delivering education and referrals for additional education and treatment.

\section{Analysis}

Descriptive statistics (means/s.d., frequencies/percentages) of chart data were conducted on quantitative chart data using Stata 13.1 (StataCorp LP, College Station, TX, USA). Summaries of weight-related discussions (free-text) were analyzed using qualitative content analysis techniques. Bivariate analyses 
( $t$-tests, correlation coefficients, $\chi^{2}$ or Fisher's exact test) were conducted to understand relationships among demographic and SCI/D characteristics, indicators of need for WM and WM treatment/recommendations. An $\alpha$-level of 0.05 was used to determine statistical significance.

\section{RESULTS}

A total of 111 charts were examined; 11 charts were excluded because the individual had died during the study period $(n=9)$ or no evidence of SCI/D was found in the chart $(n=2)$. Chart reviews were completed for 100 Veterans with SCI/D. Participants were predominantly male $(95.0 \%)$, and Caucasian $(68.0 \%)$, with an average age of 60.6 years (s.d. $=12.30$, range $=27.71-93.41$, median $=61.6$ ). On average, they had their SCI/D for 24.6 years (s.d. $=13.35$, range $=2.84-52.83$, median $=24.4$ ); $42.0 \%$ were ASIA level A and $52.0 \%$ had paraplegia. Approximately $80.0 \%$ had a spinal cord injury (versus a disorder; see Table 1).

\section{Indicators of need for WM}

Nearly three-quarters $(73.0 \%)$ of Veterans had information in their chart indicating a need for WM, including BMI of $\geqslant 25 \mathrm{~kg} \mathrm{~m}^{-2}$ $(61.0 \%)$; presence of two or more weight-related comorbidities, such as diabetes mellitus, hypertension or dyslipidemia (40.0\%); diagnosis of overweight or obesity (26.0\%); description from the provider about overweight or excess fat $(17.0 \%)$; discussion with provider of interest in WM (8.0\%); recent increase in weight $(6.0 \%)$; or recent change in function $(5.0 \%)$. When the criteria of Laughton et al. ${ }^{14}$ were used $\left(B M I \geqslant 22 \mathrm{~kg} \mathrm{~m}^{-2}\right)$, the number of Veterans with elevated BMI increased to 84 , and the total with a need for WM increased to $87.0 \%$ (see Figure 1). Average BMI of the sample was $27.7 \mathrm{~kg} \mathrm{~m}^{-2}$ (s.d. $=6.28$, range $=13.51-51.70$, median $=27.0)$. None of the charts reviewed contained any information about body fat percentage.

\section{Table 1 Participant demographic and SCI/D characteristics}

\begin{tabular}{lc}
\hline Veteran characteristics $(\mathrm{N}=100)$ & Frequency (\%) or M (s.d.) range \\
\hline Male & $95(95.0 \%)$ \\
Age (in years) & \\
Race $(\mathrm{N}=98)$ & \\
White & $68(69.3) 27.71-93.41$ \\
African American/Black & $28(28.6 \%)$ \\
Other & $2(2.0 \%)$ \\
& \\
Hispanic/Latino ethnicity & $3(3.0 \%)$ \\
& \\
Marital status & \\
Married & $40(40.0 \%)$ \\
Never married & $20(20.0 \%)$ \\
Separated/divorced/widowed & $40(40.0 \%)$ \\
& \\
Time since injury/disorder (in years) & \\
Paraplegia (vs tetraplegia) ( $N=97)$ & $52(53.6 \%)$ \\
& \\
Severity of injury (N = 93) & \\
C1-C4 AIS A, B or C & $4(4.3 \%)$ \\
C5-C8 AIS A, B or C & $23(24.7 \%)$ \\
T1-S5 AIS A, B or C & $41(44.1 \%)$ \\
Any level AIS D & $25(26.9 \%)$ \\
Traumatic etiology (vs non-traumatic) $(N=98)$ & $78(79.6 \%)$ \\
\hline
\end{tabular}

Abbreviations: AIS, American Spinal Injury Association (ASIA) impairment scale; SCI/D, spinal cord injury/disorder.
Demographic and SCI/D characteristics were examined for relationships with indicators of need for WM. Individuals with paraplegia were more likely to demonstrate a need for WM than individuals with tetraplegia $(80.8 \%$ vs $62.2 \%, P=0.04)$. No other demographic or SCI/D characteristics were associated with demonstrated need for WM. When individual indicators were examined, it was found that individuals with paraplegia were more likely to have been diagnosed with overweight or obesity $(38.5 \%$ vs $13.3 \%$, $P=0.005)$ and to have two or more weight-related conditions ( $48.1 \%$ vs $28.9 \%, P=0.05$ ). Individuals with paraplegia had, on average, higher BMIs $\left(\mathrm{M}=29.3 \mathrm{~kg} \mathrm{~m}^{-2}\right.$, s.d. $\left.=6.93\right)$ than individuals with tetraplegia $\left(\mathrm{M}=26.1 \mathrm{~kg} \mathrm{~m}^{-2}\right.$, s.d. $\left.=5.28, P=0.02\right)$. In addition, individuals with complete injuries (ASIA A) were significantly more likely to meet the criteria of Laughton et al. ${ }^{14}$ for overweight (BMI $\geqslant 22 \mathrm{~kg} \mathrm{~m}^{-2}$ ) than individuals with incomplete injuries $(90.2 \%$ vs $73.8 \%, P=0.05)$. Individuals with two or more weight-related conditions were significantly older $(M=65.9$ years, s.d. $=12.37)$ than individuals with one or no weight-related conditions $(M=57.1$ years, s.d. $=10.97, P=0.0003$ ). In addition, individuals with two or more weight-related conditions tended to have their injury/disorder longer $(M=27.6$ years, s.d. $=13.78)$ than individuals with one or no weight-related conditions $(M=22.5$ years, s.d. $=12.77)$, although this difference was only marginally significant $(P=0.06)$.

\section{WM during annual evaluations}

Seventy-five Veterans with SCI/D had progress notes available on their most recent AE; $92.0 \%(n=70)$ received a cardiovascular assessment, $86.7 \%(n=65)$ received a psychosocial assessment and $81.3 \%(n=61)$ received a rehabilitation assessment, but only $52.0 \%(n=39)$ received a nutritional assessment. Weight, if it was addressed at all during the $\mathrm{AE}$, was most likely to be discussed as a concern during the nutritional assessment $(n=25)$ as compared with the cardiovascular assessment $(n=19)$, rehabilitation assessment $(n=4)$ or psychosocial assessment $(n=1)$. Individuals with elevated BMI $\left(\geqslant 25 \mathrm{~kg} \mathrm{~m}^{-2}\right)$ were significantly more likely to receive a psychosocial assessment $(95.8 \%$ vs $70.4 \%, P=0.003)$ or a nutrition assessment $(60.4 \%$ vs $37.0 \%$, $P=0.05)$ than individuals with a BMI $<25 \mathrm{~kg} \mathrm{~m}^{-2}$. Individuals who received a psychosocial assessment also tended to weigh more $(\mathrm{M}=89.9 \mathrm{~kg}$, s.d. $=18.65 \mathrm{vs} \mathrm{M}=74.5 \mathrm{~kg}$, s.d. $=14.98, P=0.015)$ and had a higher BMI $\left(\mathrm{M}=28.5 \mathrm{~kg} \mathrm{~m}^{-2}\right.$, s.d. $=5.58$ vs $\mathrm{M}=23.2 \mathrm{~kg} \mathrm{~m}^{-2}$, s.d. $=5.07, P=0.006)$. In addition, individuals who received a nutrition assessment were also significantly more likely to be described as overweight or obese in provider progress notes ( $93.8 \%$ vs $40.7 \%$, $P<0.001)$.

During these assessments, some Veterans received recommendations with regard to WM; the most frequent recommendation during $\mathrm{AE}$ assessments was WM education $(32.0 \%, n=24)$, followed by recommendations for a healthier diet $(21.3 \%, n=16)$. Individuals who were described as overweight or obese in progress notes were more likely to be offered education ( $50.0 \%$ vs $20.3 \%, P=0.026$ ) or to receive recommendations for a healthier diet $(56.3 \%$ vs $11.9 \%, P=0.001)$. WM recommendations provided during the cardiovascular, psychosocial and rehabilitation assessments tended to be very general (for example, more physical activity). Dietitians conducting the nutrition assessment also provided recommendations on caloric and nutrient intake to four Veterans, including number of calories to consume per day, and amount of protein, carbohydrates and fluid needed (see Table 2).

Of Veterans receiving a nutritional assessment $(n=39), 92.3 \%$ $(n=36)$ received a nutrition history. None of the histories included all components recommended in the VHA Clinical Nutrition 


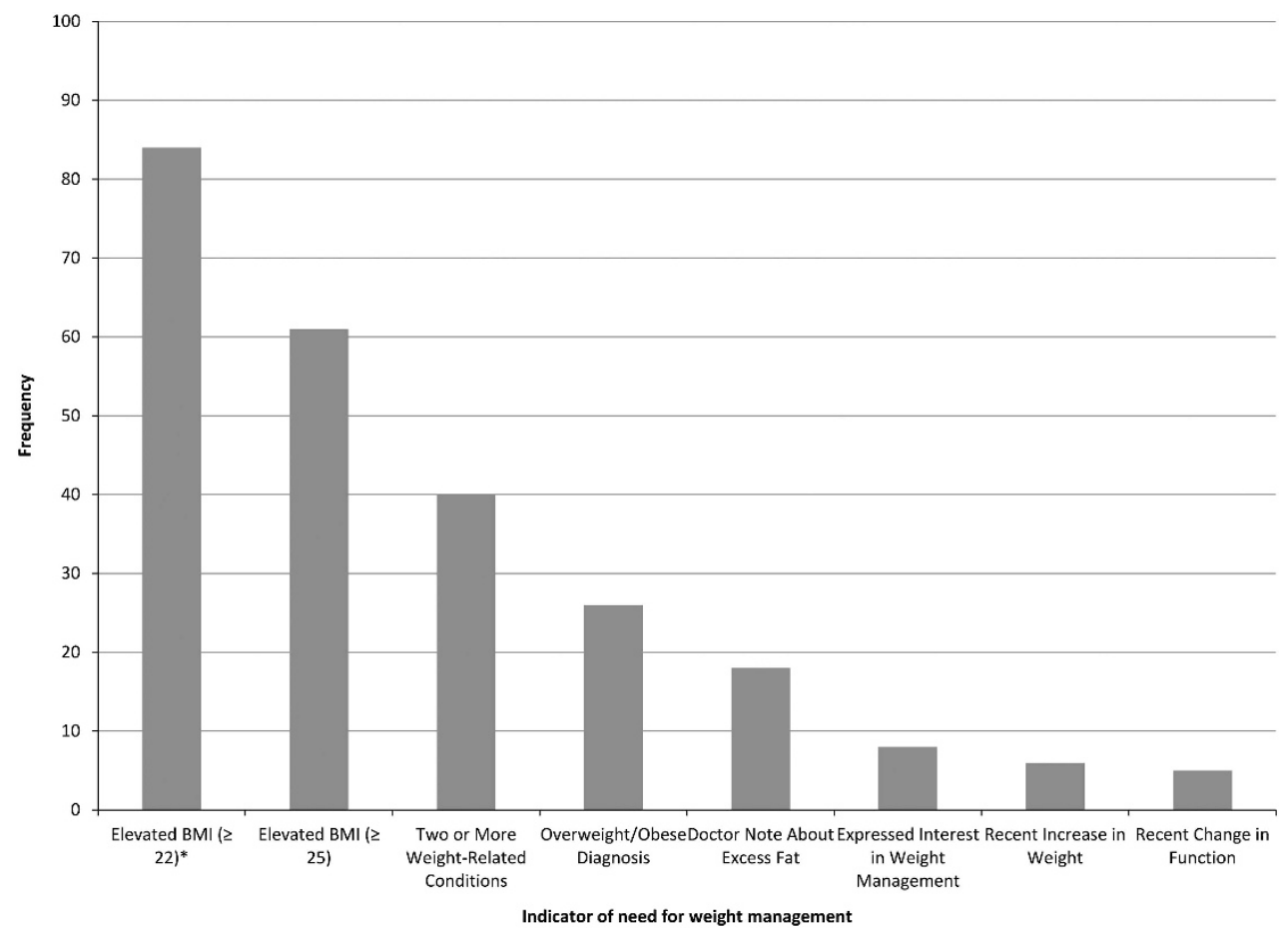

Figure 1 Frequency of indicators of need for weight management. Patients could have more than one; values do not add up to $100 \%$. ${ }^{*}$ Using the criteria of Laughton et al. ${ }^{14}$ rather than convention cutoffs.

Table 2 Weight management recommendations during annual evaluation (AE) assessments

\begin{tabular}{|c|c|c|c|c|c|}
\hline $\begin{array}{l}\text { Recommendations } \\
(\mathrm{N}=75)\end{array}$ & $\begin{array}{l}\text { Cardiovascular } \\
\text { frequency (\%) }\end{array}$ & $\begin{array}{l}\text { Psychosocial } \\
\text { frequency (\%) }\end{array}$ & $\begin{array}{l}\text { Rehabilitation } \\
\text { frequency (\%) }\end{array}$ & $\begin{array}{c}\text { Nutrition } \\
\text { frequency (\%) }\end{array}$ & $\begin{array}{c}\text { Total } \\
\text { frequency (\%) }\end{array}$ \\
\hline Healthier diet & $7(9.3)$ & $0(0.0)$ & $0(0.0)$ & $9(12.0)$ & $16(21.3)$ \\
\hline Diet prescription or nutrient support & $0(0.0)$ & $0(0.0)$ & $0(0.0)$ & $4(5.3)$ & $4(5.3)$ \\
\hline More physical activity & $6(8.0)$ & $0(0.0)$ & $0(0.0)$ & $0(0.0)$ & $6(8.0)$ \\
\hline Establish goals of treatment & $0(0.0)$ & $0(0.0)$ & $0(0.0)$ & $2(2.7)$ & $2(2.7)$ \\
\hline Education on weight management & $7(9.3)$ & $1(1.3)$ & $2(2.7)$ & $14(18.7)$ & $24(32.0)$ \\
\hline
\end{tabular}

Management Handbook. ${ }^{19}$ All or nearly all ( $n=32$ to $36,88.9$ $100.0 \%$ ) addressed feeding problems (for example, difficulty swallowing), recent weight or appetite changes and gastrointestinal problems (for example, nausea, vomiting); however, few ( $n=1$ to $3,2.8-8.3 \%$ ) addressed topics such as food intolerances, food-drug interactions or nutrient and vitamin intake, and none addressed eating disorders or unhealthy dietary behaviors (see Table 3). Individuals with recent weight gain tended to have more components covered during their nutrition history $(M=5.0$ components, s.d. $=1.15)$ than individuals without recent weight gain $(\mathrm{M}=3.9$ components, s.d. $=1.01$, $P=0.05)$. No other characteristics were related to the number of nutrition history components discussed.

WM education received outside of $\mathrm{AE}$

Outside of AEs, a total of 98 nutrition and/or WM education sessions were offered to Veterans, with 57 Veterans with SCI/D receiving education at least once, and 20 receiving such education more than once $(\mathrm{M}=1.7$ sessions, s.d. $=1.37$, range $=1-7$, median $=1)$.
Education was most frequently delivered by a nurse $(73.5 \%$ of education sessions), followed by a dietitian (12.2\% of education sessions). Individuals who were described as overweight or obese in provider progress notes were significantly more likely to receive WM education, $(82.4 \%$ vs $51.8 \%, P=0.03)$. No other characteristics or WM indicators were significantly related to receipt of education.

Two-thirds of Veterans with SCI/D receiving education were not referred for additional education or treatment, and nearly $30 \%$ refused a referral (see Figure 2). Individuals who received WM education more than once were no more likely to have been referred for additional services than individuals who received education only once (30.0\% vs $29.7 \%, P=0.983$ ).

\section{WM during inpatient care}

In addition, 25 Veterans with SCI/D had at least one inpatient stay during the previous 12 months $(M=64.7$ days, s.d. $=102.17$, range $=2-365$, median $=27$ days). The majority were weighed at least once during their inpatient stay $(72.0 \%, n=18)$, although information 
Table 3 Nutrition history components addressed during nutrition assessment

\begin{tabular}{lr}
\hline Nutrition history component $(\mathrm{N}=36)$ & Frequency (\%) \\
\hline Feeding problems (e.g., difficulty swallowing) & $36(100)$ \\
Recent weight changes & $35(97.2)$ \\
Appetite changes & $33(91.7)$ \\
Gastrointestinal problems (e.g., nausea, vomiting) & $32(88.9)$ \\
Nutrient intake & $3(8.3)$ \\
Weight history & $3(8.3)$ \\
Food intolerances & $2(5.6)$ \\
Food-drug interactions & $2(5.6)$ \\
Vitamin intake & $2(5.6)$ \\
Social background (e.g., religion, culture) & $1(2.8)$ \\
Activity level & $0(0.0)$ \\
Eating disorders & $0(0.0)$ \\
Herbal and complementary therapies & $0(0.0)$ \\
Lifestyle practices & $0(0.0)$ \\
Taste changes & $0(0.0)$ \\
Unhealthy dietary behaviora & $0(0.0)$ \\
\hline
\end{tabular}

ancludes disordered eating behaviors that may or may not meet diagnostic criteria for an eating disorder, such as binge eating, purging and so on.

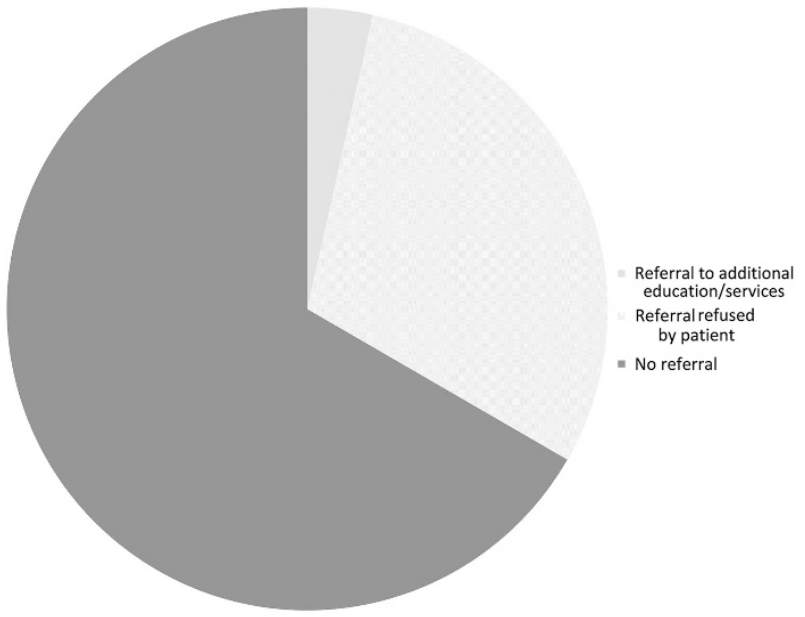

Figure 2 Referrals resulting from weight management education.

on method used to weigh the patient (for example, bed scale, Hoyer lift) was often missing. Height was never measured and, when reported $(92.0 \%, n=23)$, was almost always obtained from historic height in the patients' charts $(87.0 \%, n=20)$. Weight was discussed as a concern with only $3(12.0 \%)$ Veterans with SCI/D.

Recommendations with regard to WM included healthier diet $(n=2)$, increased physical activity $(n=1)$ and weight loss $(n=1)$; in addition, 2 Veterans with SCI/D were referred to a dietitian. More than two-thirds $(68.0 \%, n=17)$ met with a dietitian, $20(80.0 \%)$ met with a psychologist or social worker and $19(76.0 \%)$ met with a therapist (for example, physical, recreational) at least once during their inpatient stay. As with $\mathrm{AE}$, dietitians were most likely to discuss weight during these meetings $(70.6 \%, n=12)$ compared with psychologists/social workers $(10.0 \%, n=2)$ or therapists $(5.3 \%$, $n=1)$. No demographics, SCI/D characteristics or indicators of WM were significantly related to WM practices during inpatient care.

Summaries of weight-related discussions from these inpatient meetings were analyzed qualitatively; discussions involved reasons for selecting a particular inpatient diet (for example, Veterans encouraged to switch to controlled diet after frequently selecting unhealthy options on 'Vet's choice' diet), targeting irrational beliefs about WM (for example, Veterans maintain that they have lost weight despite 'what the scale says') and setting WM goals with the Veteran about nutrition $(20.0 \%, n=5)$, physical activity $(12.0 \%, n=3)$, weight loss $(8.0 \%, n=2)$ or unspecified (for example, 'discussed weight and set goals with patient,' $8.0 \%, n=2$ ).

\section{WM during outpatient care}

A total of 62 Veterans had at least one outpatient appointment with their primary care provider. Less than half were weighed at least once during these outpatient encounters $(41.9 \%, n=26)$. No variables were significant, although two were marginally significant. Individuals with a diagnosis of overweight or obesity $(78.9 \%$ vs $55.8 \%, P=0.10)$ or who met the criteria of Laughton et al. ${ }^{14}$ of overweight (BMI $\geqslant 22 \mathrm{~kg} \mathrm{~m}^{-2} ; 67.9 \%$ vs $33.3 \%, P=0.07$ ) were more likely to be weighed. Individuals who were weighed also tended to have had their injury/disorder for fewer years $(M=23.5$ years, s.d. =13.18) than individuals who were not weighed $(M=29.1$ years, s.d. $=11.28$, $P=0.09$ ).

Height was reported for slightly more than half $(54.8 \%, n=34)$, and was most frequently obtained from historic height in the chart $(79.4 \%$, $n=27)$. Only 14 Veterans $(22.6 \%)$ with SCI/D received any recommendations about WM from their provider during these appointments, including healthier diet $(n=3)$, increased physical activity $(n=3)$ and weight loss $(n=8)$. In addition, five Veterans with SCI/D were referred to the MOVE! program. Providers were more likely to discuss weight as a concern with individuals who had at least one indicator of need for WM $(31.8 \%$ vs $0.0 \%, P=0.006)$. Specific indicators that were significantly related to WM discussions were BMI $\geqslant 25 \mathrm{~kg} \mathrm{~m}^{-2}(35.9 \%$ vs $0.0 \%, P=0.001)$ and a diagnosis of overweight/obesity $(42.1 \%$ vs $14.0 \%, P=0.02)$.

Four Veterans with SCI/D met with a dietitian at least once, but only two received any recommendations about WM; one of these Veterans received recommendations on caloric and nutrient intake. Barriers to WM addressed in outpatient notes included lack of follow-up (for example, Veterans identified depression as barrier to WM with dietitian, but no evidence of follow-up by dietitian or psychologist they saw regularly) and Veteran beliefs about WM (for example, Veterans think if they exercise, they will lose essential nutrients) or misconceptions about risks from being overweight (for example, Veterans do not believe it is a risk factor for heart disease).

\section{DISCUSSION}

As found in past research, ${ }^{1,2}$ the majority of Veterans with SCI/D in the present study demonstrated a need for WM. Reducing overweight and obesity among individuals with SCI/D has the potential to increase health-related quality of life $\mathrm{e}^{11,20,21}$ and function, ${ }^{22,23}$ and decrease risk for comorbidities and complications. ${ }^{5-7}$ Overall, $73 \%$ of Veterans with SCI/D included in the study demonstrated a need for WM using conventional standards; this number increased to $87 \%$ when the standard of Laughton et al. ${ }^{14}$ was used for elevated BMI. This cutoff of $22 \mathrm{~kg} \mathrm{~m}^{-2}$ was selected because it better reflected elevated body fat percentage that, according to Laughton et al., ${ }^{14}$ is a better indicator of overweight/obesity. However, because body fat percentage was unavailable in any charts, we could not confirm the findings of Laughton et al. ${ }^{14}$ directly. In past research, ${ }^{12}$ providers were hesitant to use body fat percentage because of concerns that devices to measure body fat (body impedance analysis) were inaccurate if the patient was seated and would be difficult for individuals with limited 
arm function to use at all, and that indirect measures (for example, skin-fold thickness) were poor indicators of body fat percentage.

In the present sample, individuals with paraplegia were more likely to demonstrate a need for WM than individuals with tetraplegia. Other literature in the Veteran population has reported increased incidence of obesity among individuals with paraplegia compared with those with tetraplegia. ${ }^{24}$ Although more research is needed to explore the relationship between level of injury and need for $\mathrm{WM},{ }^{25}$ this finding provides some guidance for SCI clinicians addressing WM with their patients, particularly when counseling patients who have paraplegia.

Although level of injury did not influence WM practices, other indicators did, including BMI and visual appearance. Since the introduction of the MOVE! program, VA's electronic medical record, CPRS, reminds providers to address WM for Veterans with an elevated BMI. ${ }^{15}$ This reminder likely explains why BMI had a significant relationship with certain WM practices. Visual appearance also had a significant relationship with WM practices. Although BMI is more objective than physical appearance, researchers have noted some validity issues with using BMI as an indicator of overweight/obesity. ${ }^{26}$ Furthermore, providers have discussed concerns with BMI as an indicator of overweight and obesity in the SCI/D population. ${ }^{12}$ This finding suggests that in the absence of optimal indicators of overweight or obesity, providers may instead use visual cues to determine whether WM should be discussed with a particular patient.

There are some instances when visual cues could provide more useful information. For instance, individuals with a great deal of muscle mass would likely have a high BMI, but could be considered healthy if body fat percentage is low. In this instance, visual cues could counteract information based on BMI. In addition, increased fatty tissue on the abdomen is associated with increased risk for diabetes and cardiovascular disease, ${ }^{27}$ and may be a better indicator of health risk than BMI. $^{28}$ Once again, visual cues, such as presence of abdominal fat, may be more meaningful in this regard than overall indicators, such as BMI. However, more research is needed to determine methods for measuring overweight/obesity among individuals with SCI/D to ensure standards are consistent across patients.

VA providers have noted that weight was most frequently addressed during AEs. ${ }^{12}$ Nutrition assessments, where weight was most likely to be addressed, were also the most likely assessment to be skipped and few Veterans with SCI/D were provided with specific information on caloric and nutrient intake needs. Although a variety of websites are available to assist with determining energy expenditure and caloric needs, these calculations are based on values from able-bodied persons. Because of differences in basal metabolic rate among individuals with $\mathrm{SCI} / \mathrm{D},{ }^{10}$ individuals with $\mathrm{SCI} / \mathrm{D}$ should receive guidance on caloric and nutrient requirements from an SCI provider knowledgeable about these differences. In addition, a structured checklist or progress note would ensure that necessary elements of nutrition histories are addressed.

In contrast to AEs, weight was infrequently discussed during inpatient stays and outpatient visits. Other research conducted on this topic suggested that because of other medical needs, providers reported difficulty incorporating WM care with care for secondary conditions and complications of SCI/D. ${ }^{12}$ However, overweight/ obesity has implications for these other conditions (for example, hypertension) and complications (for example, pressure ulcers); additional work is needed to help providers incorporate WM information into care. Individuals with SCI/D frequently lack knowledge of appropriate exercise and believe exercise may be unsafe or too difficult. ${ }^{9}$ Education on exercise and physical activity appropriate for individuals with SCI/D could increase these behaviors in this population, resulting in improvements to a variety of outcomes.

Providers may also lack the knowledge and skills necessary to effectively address WM with their patients. In fact, most WM discussions, when they happened, appeared to be organized around education; there was little evidence of shared decision making or goal setting with patients based on information present in charts. Providers should be encouraged to engage in goal setting with patients wanting/needing to lose weight, rather than simply educating patients-sometimes multiple times-without any buy-in or patient engagement.

Research suggests that WM discussions are most successful when providers begin by asking patients whether they are concerned with their weight or whether they had thought about losing weight, in an empathetic non-judgmental way. ${ }^{29}$ This can lead to a discussion of how the patient could make lifestyle changes to lose weight, and what additional resources or education the patient needs to do so. Providers can draw upon the expertise of other health professionals in the SCI Center or clinic, as well as resources available through VHA and community or disability (for example, Paralyzed Veterans of America) organizations. ${ }^{12}$ Efforts to facilitate follow-up between providers would also be beneficial, particularly when a Veteran addresses a barrier with one provider (for example, dietitian) that should be addressed with another provider (for example, psychologist). Involving a patient's physician in WM counseling sessions with the dietitian may be one method to ensure follow-up and care coordination; the physician could then be responsible for involving other health-care professionals (for example, psychologists) as appropriate. ${ }^{29}$ In fact, providers in Locatelli et al. ${ }^{12}$ discussed the presence of a multidisciplinary care team as a strength of providing WM care in VA SCI Centers, because providers could call on the expertise of other health professionals.

\section{Study limitations}

The present study involved chart reviews only over a 1 -year period. As such, we may not have captured all discussions occurring during patient-provider interactions with regard to WM. However, the medical record can be considered a legal documentation of care received. In addition, we were unable to look at long-term WM practices, as well as education and other WM discussions occurring during sequential AEs. Because of sample size, we were unable to conduct statistical analyses on all practices, particularly inpatient care activities, as only 25 Veterans with SCI/D in the sample were inpatient during the study time period. A sample size of 100 was selected to allow for in-depth analysis of a small number of cases; future studies should include larger samples, and could utilize emerging analysis techniques, such as natural language processing, to extract similar data from a larger sample of cases. Because these cases were selected from three VA SCI facilities (one large and two smaller facilities), findings may not generalize to other VA SCI facilities. Furthermore, we focused on WM practices and did not examine outcomes of these practices. These findings can be used to guide studies examining the impact of practices on outcomes, to identify best practices for WM in SCI/D. Future research should also assess prescribed medications, particularly those with side effects related to appetite or weight, and determine their impact on outcomes. Because of the differences in structure of SCI care within the VA, these findings may not generalize to all patients with SCI/D not receiving treatment within the VA system. Moreover, most individuals in the present sample $(\sim 80 \%)$ had a spinal cord injury rather than a spinal cord disorder; these findings may not generalize to all individuals with spinal cord disorders. 


\section{CONCLUSIONS}

Although the majority of the Veterans with SCI/D charts indicated a need for WM, this information was inconsistently communicated to the patient. The indicators that most influenced WM discussions, treatment and education were BMI and visual cues (that is, description of the patient as overweight/obese). More research is needed to examine which indicators are most accurate among individuals with SCI/D, given past concerns with BMI. ${ }^{12,26}$ Nutrition assessments, where weight was most likely to be addressed, were the most likely assessment to be skipped during AEs. None of the nutrition histories conducted during these assessments included all of the recommended elements. ${ }^{19}$ Progress note templates may help ensure WM is being properly discussed and documented in all patient interactions, and that nutrition histories are complete. Few Veterans with SCI/D were provided with specific information on caloric and nutrient intake needs. Weight was infrequently addressed during outpatient visits or inpatient stays. Additional work is needed to help providers to incorporate WM information into care. The present research also found some evidence of irrational beliefs and misinformation among Veterans with SCI/D that could be remedied by SCI/D and/or WM providers addressing these topics. As such, it is essential that providers work to educate about the importance of WM.

\section{DATA ARCHIVING}

There were no data to deposit.

\section{CONFLICT OF INTEREST}

The authors declare no conflict of interest.

\section{ACKNOWLEDGEMENTS}

Research was funded by the Department of Veterans Affairs Health Services Research \& Development Quality Enhancement Research Initiative (QLP 97-006, PI: LaVela, Co-PI: Locatelli). The opinions expressed in this manuscript are the views of the authors and do not necessarily represent the views of the Department of Veterans Affairs or United States Government. This study was presented at the meeting of the Academy of Spinal Cord Injury Professionals, September 2015, New Orleans, LA, and received a Trainee Research Award. In addition, the abstract of this presentation was published in the September 2015 issue (Volume 38, Issue 5) of the Journal of Spinal Cord Medicine.

1 De Groot S, Post MWM, Postma K, Sluis TA, van der Woude LHV. Prospective analysis of body mass index during and up to 5 years after discharge from inpatient spinal cord injury rehabilitation. J Rehabil Med 2010; 42: 922-928.

2 Groah SL, Nash MS, Ward EA, Libin A, Mendez AJ, Burns P et al. Cardiometabolic risk in community-dwelling persons with chronic spinal cord injury. J Cardiopulm Rehab Prev 2011; 31: 73-80.

3 Guh DP, Zhang W, Bansback N, Amarsi Z, Birmingham CL, Anis AH. The incidence of co-morbidities related to obesity and overweight: a systematic review and meta-analysis. BMC Public Health 2009; 9: 88

4 Nyland J, Quigley P, Huang C, Lloyd J, Harrow J, Nelson A. Preserving transfer independence among individuals with spinal cord injury. Spinal Cord 2000; 38: 649-657.
5 Elsner JJ, Gefen A. Is obesity a risk factor for deep tissue injury in patients with spinal cord injury? J Biomech 2008; 41: 3322-3331.

6 Chen Y, Cao Y, Allen V, Richards JS. Weight matters: physical and psychosocial well being of persons with spinal cord injury in relation to body mass index. Arch Phys Med Rehabil 2011; 92: 391-398.

7 Galea MP. Spinal cord injury and physical activity: preservation of the body. Spinal Cord 2012; 50: 344-351.

8 Barfield JP, Malone LA. Perceived exercise benefits and barriers among power wheelchair soccer players. J Rehabil Res Dev 2013; 50: 231-238.

9 Cowan RE, Nash MS, Anderson KD. Exercise participation barrier prevalence and association with exercise participation status in individuals with spinal cord injury. Spinal Cord 2013; 51: 27-32.

10 Price M. Energy expenditure and metabolism during exercise in persons with a spinal cord injury. Sports Med 2010; 40: 681-696.

$11 \mathrm{Kehn} \mathrm{M,} \mathrm{Kroll} \mathrm{T.} \mathrm{Staying} \mathrm{physically} \mathrm{active} \mathrm{after} \mathrm{spinal} \mathrm{cord} \mathrm{injury:} \mathrm{a} \mathrm{qualitative}$ exploration of barriers and facilitators to exercise participation. BMC Public Health 2009; 9: 168.

12 Locatelli SM, Gerber BS, Goldstein B, Weaver FM, LaVela SL. Health care provider practices, barriers, and facilitators for weight management for individuals with spinal cord injuries and disorders. Top Spinal Cord Inj Rehabil 2014; 20: 329-337.

13 Buchholz AC, Bugaresti JM. A review of body mass index and waist circumference as markers of obesity and coronary heart disease risk in persons with chronic spinal cord injury. Spinal Cord 2005; 43: 513-518.

14 Laughton GE, Buccholz AC, Martin Ginis KA, Goy RE. Lowering body mass index cutoffs better identifies obese persons with spinal cord injury. Spinal Cord 2009; 47: 757-762.

15 Kinsinger LS, Jones KR, Kahwait L, Harvey R, Burdick M, Zele V et al. Design and dissemination of the MOVE! weight-management program for Veterans. Prev Chronic Dis 2009; 6: A98.

16 Weiner BJ, Haynes-Maslow L, Kahwati LC, Kinsinger LS, Campbell MK. Implementing the MOVE! weight management program in the Veterans Health Administration, 2007-2010: a qualitative study. Prev Chronic Dis 2012; 9: E16.

17 US Department of Veterans Affairs VHA handbook 1176.01: Spinal cord injury and disorders (SCI/D) system of care. February 2011 http://www.va.gov/vhapublications/ ViewPublication.asp?pub_ID =2365 (Accessed on 2 July 2015).

18 Collins EG, Langbein WE, Smith B, Hendricks R, Hammond M, Weaver F. Patients' perspectives on the comprehensive preventive health evaluation in veterans with spinal cord injury. Spinal Cord 2005; 43: 366-374.

19 US Department of Veterans Affairs. VHA handbook 1109.02: Clinical nutrition management. February 2012 http://www.va.gov/vhapublications/ViewPublication.asp? pub_ID = 2493 (Accessed on 2 July 2015).

20 Mann PJ, Chad KE. Components of quality of life for persons with quadriplegic and paraplegic spinal cord injury. Qual Health Res 2001; 11: 795-811.

21 Noreau L, Shephard RJ. Spinal cord injury, exercise and quality of life. Sports Med 1995; 20: 226-250.

22 Chen Y, Henson S, Jackson AB, Richards JS. Obesity interventions in persons with spinal cord injury. Spinal Cord 2006; 44: 82-91.

23 Ragnarsson KT. Functional electrical stimulation after spinal cord injury: current use, therapeutic effects and future directions. Spinal Cord 2008; 46: 255-274.

24 Weaver FM, Collins EG, Kurichi J, Miskevics S, Smith B, Rajan S et al. Prevalence of obesity and high blood pressure in Veterans with spinal cord injuries and disorders: a retrospective review. Am J Phys Med Rehabil 2007; 86: 22-29.

25 Rajan S, McNeely MJ, Warms C, Goldstein B. Clinical assessment and management of obesity in individuals with spinal cord injury: a review. J Spinal Cord Med 2008; 31 : 361-372.

26 Romero-Corral A, Somers VK, Sierra-Johnson J, Thomas RJ, Collazo-Clavell ML, Korinek $\mathrm{J}$ et al. Accuracy of body mass index in diagnosing obesity in the adult general population. Int J Obesity 2008; 32: 959-966.

27 Björntorp P. 'Portal' adipose tissue as a generator of risk factors for cardiovascular disease and diabetes. Arterioscler Thromb Vasc Biol 1990; 10: 493-496.

28 Janssen I, Katzmarzyk T, Ross R. Waist circumference and not body mass index explains obesity-related health risk. Am J Clin Nutr 2004; 79: 379-384.

29 Rao G, Burke LE, Spring BJ, Ewing LJ, Turk M, Lichtenstein AH et al. New and emerging weight management strategies for busy ambulatory settings: a scientific statement from the American health Association endorsed by the Society of Behavioral Medicine. Circulation 2011; 124: 1182-1203. 